JIM

ISSN: 2183-0606

(CC BY 3.0)

Vol. $9,4(2021)$

p. $80-97$

AM: Jul $/ 2021$

SM: Jun $/ 2020$

\title{
Building Pillars Where Bridges are Easy to Imitate - Mediating Technologies in Knowledge-Based Economies
}

\author{
Guido Fioretti ${ }^{1}$, Stephen K. Kwan ${ }^{2}$, and Stefania Niciforo ${ }^{3}$ \\ ${ }^{1}$ University of Bologna. Via Zamboni, 33 - 40126 Bologna, Italy | guido.fioretti@unibo.it \\ ${ }^{2}$ San José State University, 1 Washington Sq, San Jose, CA 95192, USA | stephen.kwan@sjsu.edu \\ ${ }^{3}$ University of Bologna. Via Zamboni, 33 - 40126 Bologna, Italy | stefania.niciforo@gmail.com
}

\begin{abstract}
We carried out a qualitative investigation of a pioneer of the Industrial Internet of Things operating in Silicon Valley since 1989. We reconstructed a sequence of strategic turns and conceptualized their current business model in a way that is relevant for several other industries enabled by the Internet, generalizing Thompson's concept of mediating technologies in order to adapt it to contemporary, knowledge-based economies. We propose an interpretive framework for mediating technologies that we express by means of semantic networks.
\end{abstract}

Keywords: Industrial Internet of Things; Knowledge Management; Digital Technologies; Mediating Technologies.

Cite paper as: Fioretti, G., Kwan, S.K., Niciforo, S., (2021). Building Pillars Where Bridges are Easy to Imitate Mediating Technologies in Knowledge-Based Economies, Journal of Innovation Management, 9(4), 80-97.; DOI: https://doi.org/10.24840/2183-0606_009.004_0005

\section{Introduction}

The Internet made information available so easily, quickly and often at zero cost, that "business as usual" has become impossible in quite many established industries ranging from music to news. Many firms have been forced to re-think the assumptions of their strategies, asking themselves what competencies would produce value and, most importantly, how they could harness it. In some cases, their whole business model had to change and novel sources of value had to be found (Lumpkin and Dess, 2004).

In quite many cases, the new emerging business model consists of providing services or enhanced features for goods that cannot be sold because they are already available for free. A classical instance are firms selling installation and maintenance services for free software, but examples can be found in mature industries, too (Teece, 2010).

At a closer scrutiny, this class of business models applies to a specific class of technologies. In a classical taxonomy, Thompson (1967) traced a distinction between long chain technologies for mass production of standardized goods, intensive technologies for delivering customized services implying substantial customer feedback, such as those provided by healthcare organizations, and mediating technologies that connect two otherwise disconnected populations. In general, firms that are still operating long chain technologies have not been heavily affected by the Internet. On the other hand, the business model of vendors of customized services for free software is typical of 
intensive technologies because it is based on extensive feed-backs with their clients. In quite many cases, the Internet revolutionized the business models of many intensive technologies, opening up possibilities for extracting value from providing assistance, maintenance and feed-back. There remains the question of what happened to mediating technologies.

According to Thompson (1967), mediating technologies are those that connect populations who wish to be interdependent from one another. Examples of organizations based on mediating technologies include commercial banks linking lenders and borrowers as well as insurance companies linking those who wish to pool risks. Certain businesses that are based on mediating technologies did not change their nature although they have been immensely impacted by the Internet e.g., customer-to-customer petty commerce of used goods changed a lot since it moved from small local newspapers to web sites, but insofar it connects local traders it's essentially the same business (Dhanorkar, 2019). In other cases a radically novel element entered the picture, one that Thompson could not envisage.

Consider search engines. Since they connect pieces of information that are available on the Internet, they are clearly based on mediating technologies. However, web sites are freely available to all search engines. Thus, search engines can only differentiate from one another by the algorithms that they employ in order to uncover relevant items. Likewise, in principle all social communication media can connect all phone numbers in the world, but they differ by the rules by which they create online communities. In these examples, mediating technologies have been enriched by the ability to uncover or create structures within the populations that they connect. This is of utmost importance, because whenever information becomes available for free, bridging populations becomes easy to the point of not providing any value anymore. By contrast, by uncovering or creating structures in networks that support their ability to bridge otherwise disconnected items, firms can build pillars that may be difficult to imitate. Our point is that, with information available for free, the business models that are appropriate for mediating technologies may rest to a greater extent on pillars than on the bridges that these technologies build.

We would not contend that in the pre-Internet era companies based on mediating technologies would never care about the pillars that enabled them to bridge otherwise disconnected populations. Albeit the structure of networks of connections in the served populations possibly did not matter to large commercial banks or general-purpose insurance companies, it has always been important for local savings banks or merchant banks, just to name a couple of different examples in the same industry (Engelberg et al., 2012). Nevertheless, we suggest that when bridging becomes all too easy to imitate, pillars becomes key to obtain a competitive advantage. In our opinion, this development created a whole new class of business models that enriched and qualified Thompson's (1967) definition of mediating technologies.

We discovered this class of business models while investigating a Silicon Valley firm that operates in the Industrial Internet of Things (IloT), pioneering this field since the 1990s. Discussing this case-study is useful in order to add some flesh to the above concepts.

The rest of this paper is organized as follows. In the ensuing section (2) we introduce our case-study explaining the features and potentialities of the Internet of Things (loT) and the Industrial Internet of Things (IloT) for the firm that we investigated. Section (3) illustrates our methodology. Section (4) reconstructs the strategies that this firm explored and illustrates its current business model. Section (5) interprets this business model in terms of mediating technologies and frames it by means of semantic networks. Section (6) discusses implications for businesses and scholars. Finally, Section (7) concludes. 


\section{Distributed Control, IoT and IloT}

The Internet has created unprecedented possibilities by connecting millions of computers in a huge web where information fluxes - unlike many previous communication media - are neither planned, nor centralized. As early as in the 1980s, at a time when the Internet was still in its infancy, a few visionary IT specialists speculated that if microchips would be embedded in physical devices that could communicate with one another, their functionalities would go well beyond those of each single device (Wired, 1993). For example, an occupancy sensor, a light switch and a thermostat could regulate the lighting and temperature of a room as soon as someone entered it. Or, an alarm clock could wake you up but also send a message to a machine that brews a cup of coffee. Similar applications would span as diverse fields as building automation, agile productive plants, the grid of power plants or, in short, any setting where a large number of heterogeneous devices must coordinate their operations while retaining some degree of autonomy.

In 1988, a few prominent Silicon Valley venture capitalists and entrepreneurs founded a company named Echelon in order to bring this vision to market. Echelon designed the Neuron, a microchip that could be embedded in physical devices which, in their turn, would be connected to one another through the power line network (PLN). Echelon would sell the Neuron and the LonTalk communication protocol, which together made up the LonWorks control technology (Lane and Maxfield, 2005, 2009).

The architecture designed by Echelon was radically different from the leading technological paradigm of those times. According to the prevailing wisdom of the 1980s and 90s, sensors and actuators would be wired through a hierarchical set of data hubs which would route all information to a central computer, which in its turn, would issue appropriate commands to local devices. By contrast, Echelon set out to implement a distributed control architecture. Each device would be endowed with its own microchip, and each device would be able to communicate with any other without any need for centralized control (Lane and Maxfield, 2005).

Distributed control is technically superior to centralized control. Its advantages are due to (i) scalability, in the sense that devices can be added or taken away without re-programming the whole system, and (ii) reliability in case of power shortages, for several microchips working in parallel take a much shorter time to restore normal operation. From a purely technical point of view, there was no doubt that distributed control would make centralized control obsolete (Casalini et al., 2016).

However, distributed control provides its biggest benefits if a large number of heterogeneous devices are connected, which are eventually produced by competing firms. Each of these firms may have an interest in connecting its own devices to one another by means of a distributed architecture, but none of them is happy to see distributed networks where devices from different vendors can be employed and therefore compete with one another. Each producer in each industry strives to sign contracts requiring that only its own products are used, and each producer typically markets devices that are not easily compatible with those of its competitors. Since distributed control is unlikely to display its full potentialities if it is applied to devices of one maker only, we are experiencing the paradoxical reality of a superior technology that is technically available since the end of the 1980s, but has not been able to spread into the economy hitherto (Rossi et al., 2009).

Distributed control did not spread, but it did not disappear either. As a matter of fact, it survived and diffused in specific market niches. Echelon did not thrive, but it did not go bankrupt either. It rather set on a slow but steady growth path, possibly disappointing with respect to the initial (exaggerated) expectations but respectably positive and reasonably good in the long run. 
Quite surprisingly for the disillusioned fans of distributed control, the new millennium brought a new spike of expectations. This new wave of applications also had a brand-new name: The Internet of Things, which would connect any sort of devices making them smart. Remarkably, this new name did not stress technical superiority (distributed control vs. centralized control) but rather what the devices would do for the final user: They would provide environments where devices would connect to one another just like computers do.

This focus on consumers is possibly connected with an afterthought that is likely to loom very large in the minds of all actors involved, namely, the possibility that "smart" devices are used to collect big data about consumer habits. If this will ever come true, then distributed control will finally spread, not because of its technical superiority but rather because the benefits of profiling consumers may outweigh the costs of greater competition between producers of smart devices (Ziegeldorf et al., 2013).

Interestingly, Echelon is staying away from all issues regarding big data and loT. It is focusing on the 'Industrial' loT instead (IloT), where technical expertise matters and where profits can be made here and now rather than at some distant future. In retrospect, one may observe that throughout its whole existence Echelon stayed firmly within the IloT although since the 1990s it switched its main focus from building automation to power grids, and later on to lighting systems (Echelon, 2014). In broad terms, we can observe a certain consistency with its origins (Hannan and Freeman, 1984) co-existing with the entrepreneurial ability of re-directing core activities towards a series of different markets.

\section{Methodology}

We were initially attracted to the Echelon story because we were impressed by the fact that a potentially successful innovation could not diffuse because of established market structures (Lane and Maxfield, 2005). Moreover, we were intrigued by the fact that, showing a perfectly ecological pattern, this technology neither thrived nor disappeared but rather survived in a series of small niches to which it adapted while retaining its core features (Kemp et al., 1998).

Two extensive academic investigations had been already carried out by partially overlapping research teams, in 1996-98 (Lane and Maxfield, 2005) and 2003-2005 (Lane and Maxfield, 2009; Rossi et al., 2009), respectively. ${ }^{1}$ We obtained the materials that they collected, which we used as a starting point for our own investigation. We approached Echelon in 2015, carrying out open-ended interviews that aimed at discovering how the creation of a market for distributed control was eventually going on. Our claims refer to 2015.

We carried out a qualitative research following the guidelines of "Glaser's", or "Classical" Grounded Theory (CGT) (Glaser and Strauss, 1967; Glaser, 1992; Orton, 1997). It is worth stressing that, out of the various flavours of Grounded Theory, CGT is the one that least relies on pre-determined conceptual schemes and codified procedures for data collection and processing (Walker and Myrick, 2006; Evans, 2013). In particular, CGT rejects or at least discourages any usage of techniques such as questioning or text analysis on the ground that they force the data into preconceived schemes. CGT is based on repeated analysis and comparison of documents and notes inducing the generation and observation of theoretical constructs at growing levels of abstraction (Glaser, 2002; Glaser and Holton, 2004). Reaching theoretical statements out of analysis of qualitative data is its goal and measure for success (Suddaby, 2006). In our case, we

1. The first team was not allowed to publish all materials they had collected, which they eventually returned to Echelon

http://www.open-jim.org 
moved away from the initial intention to investigate the interplay between change and continuity in Echelon's strategy to derive general statements concerning business models in industries that are heavily affected by ICTs.

During our investigation we made open-ended interviews with the following Echelon officials: ${ }^{2}$

- Chris Dingley, VP for Sales (unrecorded);

- Robert Dolin, former CTO and VP for Engineering (recorded);

- Robert Maxfield, Bord Member since inception (recorded);

- Sohrab Modi, CTO and VP for Engineering (unrecorded);

- Ronald Sege, CEO and Chairman of the Board (unrecorded).

Handwritten notes were taken in all interviews, including those where recording was not allowed. Excerpts from these notes were submitted to interviewees for approval.

One of us attended the following debates in Silicon Valley on IoT, IloT and big data:

- InnoWest 2015 Conference;

- IEEE SIIT (Standardization and Innovation in linformation Technologies) 2015;

- IBM Watson: Expand Watson Platform for Next Generation of Builders, Extend Industry's Largest Portfolio of Cognitive APls, 2015;

- Samsung Sami Platform Powering the loT, 2015;

- Google: Robotics/loT Using Go, 2015.

In particular, we were struck by a scheme proposed at the InnoWest Conference by a specialized consultant, Claro Partners (2014). Our key conceptual advance occurred when we combined Claro Partners' insights with the inputs provided by Echelon to extract the features of their business model. Subsequently, we generalized our interpretive framework.

In detail, we went through the following steps:

1. We approached Echelon with a desire to investigate how the creation of a market for distributed control was proceeding, niche by niche or otherwise. We were knowledgeable about Echelon's past unexpected difficulties in spite of being the pioneer of a superior, potentially path-breaking technology;

2. We found a fascinating evergreen start-up that was continuously reinventing itself, boldly facing its second strategic turn towards smart lighting after having abandoned smart grids, which had supersed building automation in their turn;

3. We mapped Echelon's understanding of itself onto Claro Partner's scheme of loT/IloT (reproduced in Figure (2)) as bridging between the physical and the digital world, checking the robustness of this conceptual mapping with Echelon officials;

4. We generalized this interpretive scheme, connecting it to Thompson's concept of mediating technologies while updating it to contemporary knowledge-based economies;

5. We expressed our scheme by means of semantic networks, which allow to illustrate the structure of knowledge pillars on which bridges can eventually rest;

6. We distilled propositions that capture and summarize our findings, suggesting that our interpretive scheme may turn out useful in other contexts.

Among the above steps, (3), (4) and (5) were the most important ones. First of all, (3) ignited our whole chain of reasoning. However, it was step (4) that enabled us to explain what we were doing by embedding our discourse within existing theories. Finally, step (5) allowed us to stress the importance of knowledge structures. Step (5) is important because the clarity provided by semantic networks may possibly prelude to semi-quantitative investigations of similar business models.

2. We always asked for permission to record interviews. In four out of six interviews, permission was not granted. 
The sequence of conceptual steps by which we arrived at our interpretation does not always correspond to our presentation. In particular, we chose to mention Thompson at the very beginning in order to ease framing with existing theories.

Finally, it is worth stressing that our interpretation of Echelon's business model does not necessarily coincide with Echelon's interpretation of facts. It is our own theory, grounded on testimonies provided by Echelon as well as other sources (Glaser, 2002).

\section{Change and Continuity Towards a Business Model}

Echelon was founded in 1988 in order to provide distributed solutions to control problems affecting as diverse industries as manufacturing, building automation, consumer durables and beyond (Echelon, 1990). Management had to make a choice. At that time, it seemed reasonable to begin with a section of building automation where the potential advantages from connecting devices to one another were substantial, namely, Heating, Ventilation, and Air Conditioning industries (HVAC).

Echelon started selling its microchips in 1990. Its initial strategy consisted of stipulating agreements with the main HVAC manufacturers in order to insert microchips in their devices. Since 1996, a new strategy complemented these agreements (Lane and Maxfield, 2005). According to this new strategy, Echelon would produce modules endowed with Neuron chips that could interface with devices of any maker. These general-purpose modules would be sold to independent 'system integrators' who would in their turn provide solutions incorporating devices from multiple suppliers.

A new turning point occurred in 2000, when the Italian electricity provider Enel asked Echelon to add its microchips to a new series of its meters. Plans were ambitious, for Enel's CEO aimed at delivering solutions for home automation through its partnership with Echelon (Tatò, 2016). This agreement brought about a strategic turn in the long run. Within a few years, although applications to building automation were still being made (through system integrators as well as through agreements with HVAC manufacturers), power grids had become Echelon's main focus.

Unfortunately, it turned out that most electricity providers had little interest in combining intelligent meters with home automation. Most of them were just interested in remote reading, a basic functionality for which cheaper devices were available (Thoma, 2017). Even Enel lost interest in integrating home automation with their metering network when a new CEO was appointed in 2002 (Dolin, 2015). For a few years Echelon continued to pursue the strategy that Enel had suggested, but in 2014 it eventually sold its smart grid operations in order to enter smart lighting through the acquisition of Lumewave (Echelon, 2014). Throughout this transition its discourse shifted from "distributed control" to IloT.

Echelon was founded with the idea of providing a general solution to control problems in any industry but, in practice, it could only zig-zag across separate markets such as HVAC, power grids and lighting (Lane and Maxfield, 2009). What is most remarkable with these strategy shifts is that in spite of all the changes, and in spite of all the hype surrounding loT and big data, Echelon never deviated from understanding itself as an organization based on technical excellence. Apparently, it has never been tempted by the possibility of using smart devices in order to enter the business of consumer profiling. On the one hand, its zig-zag through strategies and markets is an instance of change management. On the other hand, at a more fundamental level that defines its identity and the source of its competitive advantage, Echelon appears not to have ever changed (Hannan and Freeman, 1984).

Across so many shifts in strategy, Echelon has always been looking for a suitable business 
model in an industry where profits are still rare. In general, experts claim that the added value of the IloT will arise out of a combination of hardware, software and consumer semantics. In a nutshell, experts generally claim that smart devices can add logical capabilities to physical signals but functionalities that have a meaning for the final user can only emerge when devices are networked to one another (Yoo et al., 2010; Claro Partners, 2014; Fleisch et al., 2014).

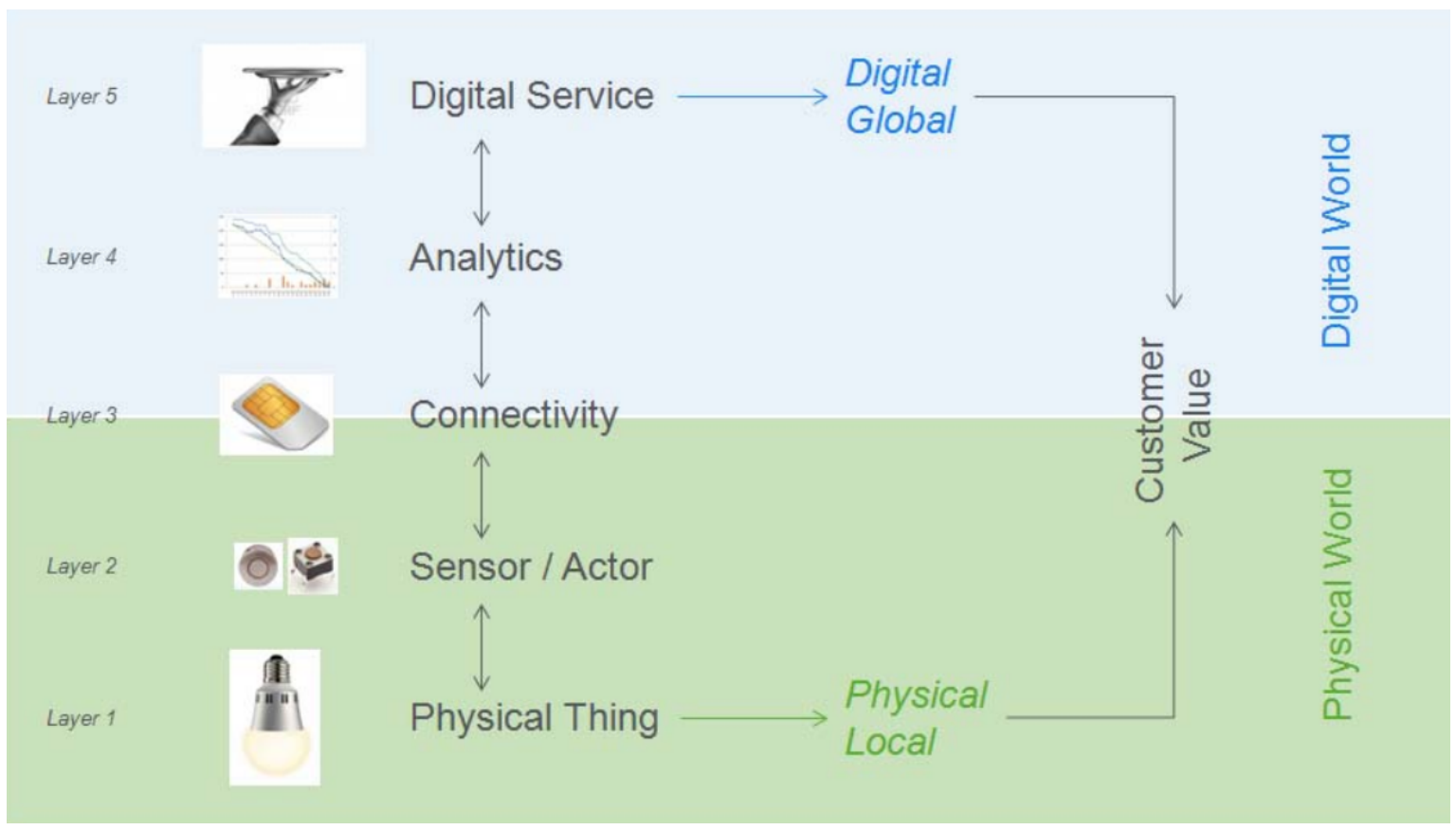

Figure 1. (By courtesy of Fleisch et al., 2014). Customer value is supposed to arise at the interface between the physical and the digital world. Bi-directional arrows between functionality layers mean that each layer should be designed in conjunction with the neighboring ones.

Figures 1 and 2 reproduce two schemes proposed in the literature. Physical signals (hardware) and logical capabilities (software) are pillars over which a link or bridge can be stretched, that yields customer value (semantics). The meaning of these schemes becomes clear by applying them onto Echelon's business model.

In its early days, Echelon sold microchips that would communicate with one another through the proprietary protocol LonTalk. In its early view, Microsoft was the example to imitate. Echelon dreamed of a near-monopolistic position where its proprietary communication protocol LonTalk would play a role similar to the Windows operative system. This dream never realized because - unlike the duopoly of Apple and Microsoft in the personal computer market - quite many producers of smart devices existed, none of whom wanted a standard to exist (Lane and Maxfield, 2005).

Today, Echelon makes use of an open communication protocol (Thoma, 2017). Instead of making profits by selling the real thing (i.e., the Neuron microchip, the LonTalk communication protocol), it generates revenue by designing IloT solutions for specific customers. However, its IloT solutions differentiate themselves from those of most competitors.

In the 1990s and 2000s, Echelon implemented distributed control using power line communication (PLC). PLC is based on the fact that since electrical signals of different frequencies can travel on the same wire without confusing one another, the wires that host the usual $220 \mathrm{~V}$ alternate current at a frequency of $50-60 \mathrm{~Hz}$ can also be used to make sensors and actuators communicate 


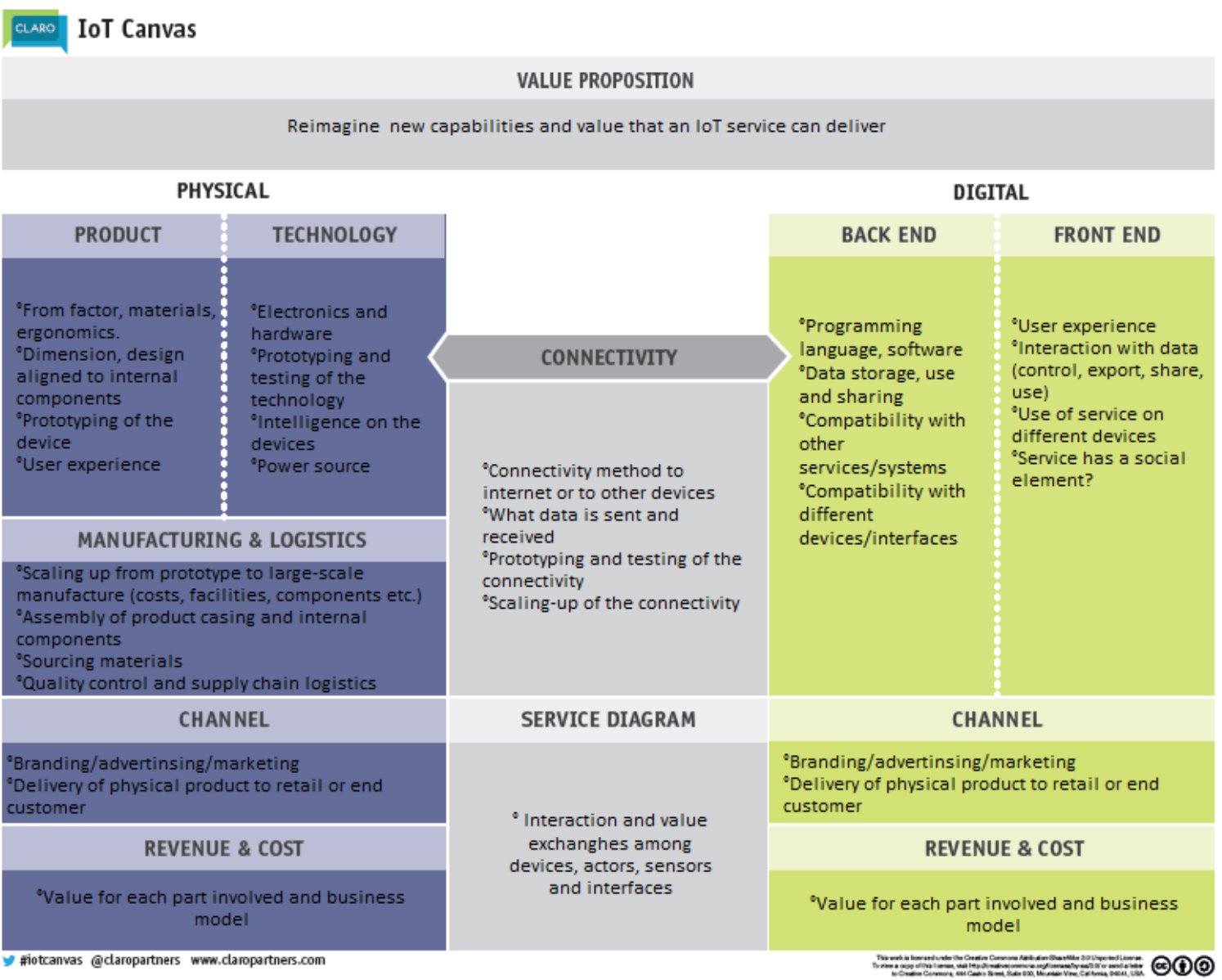

Figure 2. (Claro Partners, 2014. Creative Commons BY-NC-SA 3.0). Customer value is supposed to arise from the ability to connect the physical world to the digital world.

with one another. Today, most loT applications are based on wireless communication (WC) because it is cheaper and easier to set up, but both technologies have advantages and drawbacks. On the one hand, WC is difficult or impossible if there are obstacles such as tunnels or large buildings. On the other hand, PLC has trouble getting around transformers. Furthermore, its cost is typically higher than WC.

Therefore, Echelon is currently proposing arrangements that combine WC with PLC in order to provide more comprehensive solutions. Customer value is provided by device connectivity, and connectivity is ensured by the combination of WC and PLC. In terms of the above schemes (Claro Partners, 2014; Fleisch et al., 2014), one of the pillars, the hardware, entails knowledge that is difficult to imitate. The knowledge represented by the other pillar is the software, which is available for free. And the bridge, the semantics yielding meaningful customer services, is generated by connections between devices that can be easily arranged by anyone.

Figure 3 illustrates Echelon's current business model. The bridge generates valuable customer value, but it must be given away for free because the attempt to protect it by means of a proprietary protocol failed. The source of revenues is one element in the technical pillar on the left, namely PLC expertise. 


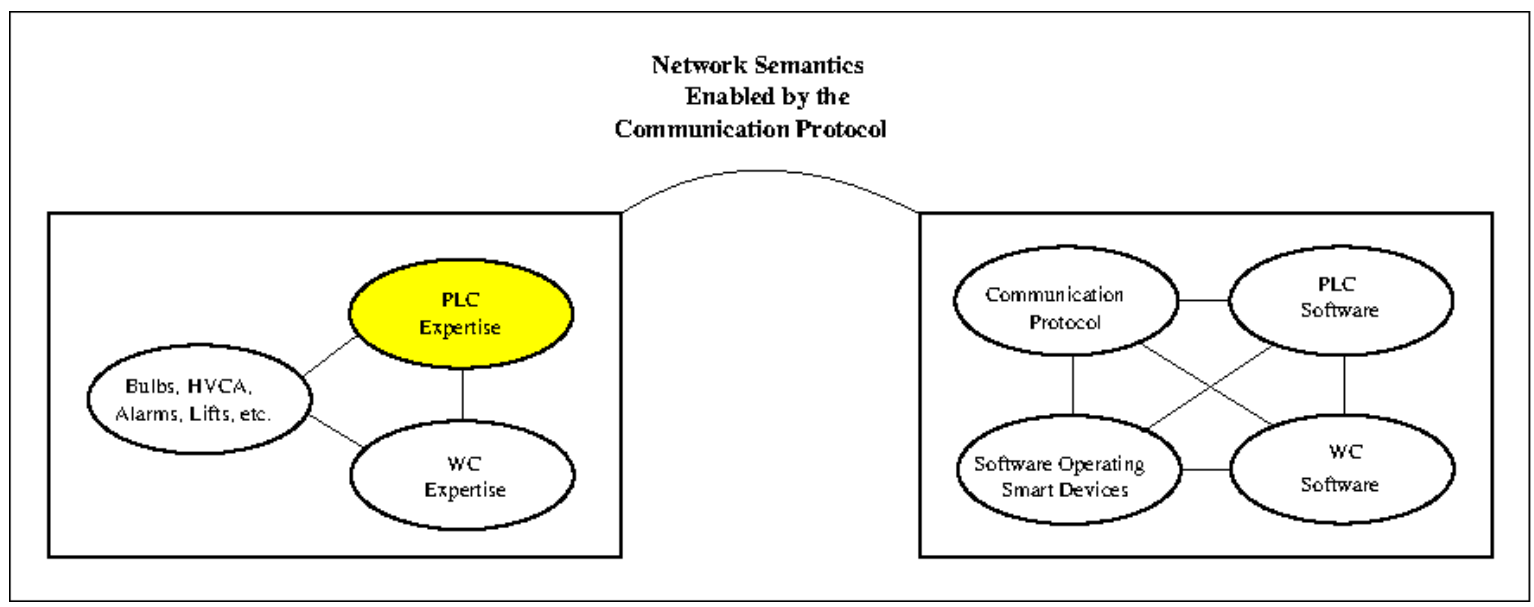

Figure 3. Echelon's current business model (2015). On the left, technical knowledge about devices as well as WC and PLC. On the right, knowledge about software and communication protocols. Customer value is generated by the bridge in-between, but only PLC expertise is difficult to imitate.

\section{Semantic Network Analysis}

Semantic networks are bipartite graphs where nodes represent concepts linked to one another by logical relations represented by edges. In the simplest versions, edges represent definitions or assertions (Sowa, 1992).

We propose to make use of semantic networks in order to illustrate the knowledge structure of business models such as Echelon's. In particular, semantic networks allow to illustrate the structure of the pillars that enable conceptual bridges such as those built by Echelon.

In our usage of semantic networks, nodes represent pieces of knowledge. They are connected to one another if they are sufficiently similar to be properly communicated and understood, and yet at the same time sufficiently different to be interesting to one another. In other words, two pieces of knowledge must be at some intermediate cognitive distance from one another in order to be linked to one another in our semantic networks (Nooteboom, 2000). For instance, the fact that Echelon fruitfully combines WC with PLC appears as an edge between nodes representing these two pieces of knowledge.

In general, an organization's knowledge on a certain domain is unlikely to be represented by one node only. For instance, knowledge about PLC is likely to be composed by a number of concepts, represented by a node each. However, the level of detail of our sketched semantic networks will not be realistic. Any degree of aggregation will be allowed, with nodes possibly representing bodies of knowledge that in reality entail a large number of concepts. Therefore, we generically refer to nodes as representing pieces of knowledge, rather than concepts.

Let us import a distinction between weak ties and strong ties that has originally been conceived for social networks (Burt, 1992). Let us stipulate that, in semantic networks, strong ties tightly connect pieces of knowledge that are permanently combined in organizational knowledge. By contrast, weak ties link pieces of knowledge that are occasionally connected to one another, in qualitatively different ways. For instance, the ability to combine WC with PLC is distinctive of Echelon's knowledge. In their knowledge base, this is a permanent, strong tie. By contrast, every single contract requires a new bridge to figure out which devices should be employed and connected to one another. This is an ad hoc, volatile tie. Let us stipulate that, in our framework, bridges are weak ties whereas pillars are made of strong ties. 
A semantic network can capture business models such as Echelon's by means of two sets of nodes representing knowledge that pertains to different domains, physical signals and software, respectively. Echelon is eventually able to bridge between these two domains, generating customer value.

The left side of Figure 4 illustrates this sort of situations in more general terms. Blue nodes represent pieces of knowledge pertaining to two domains. A firm owns pieces of knowledge highlighted in red that enable it to bridge between them.

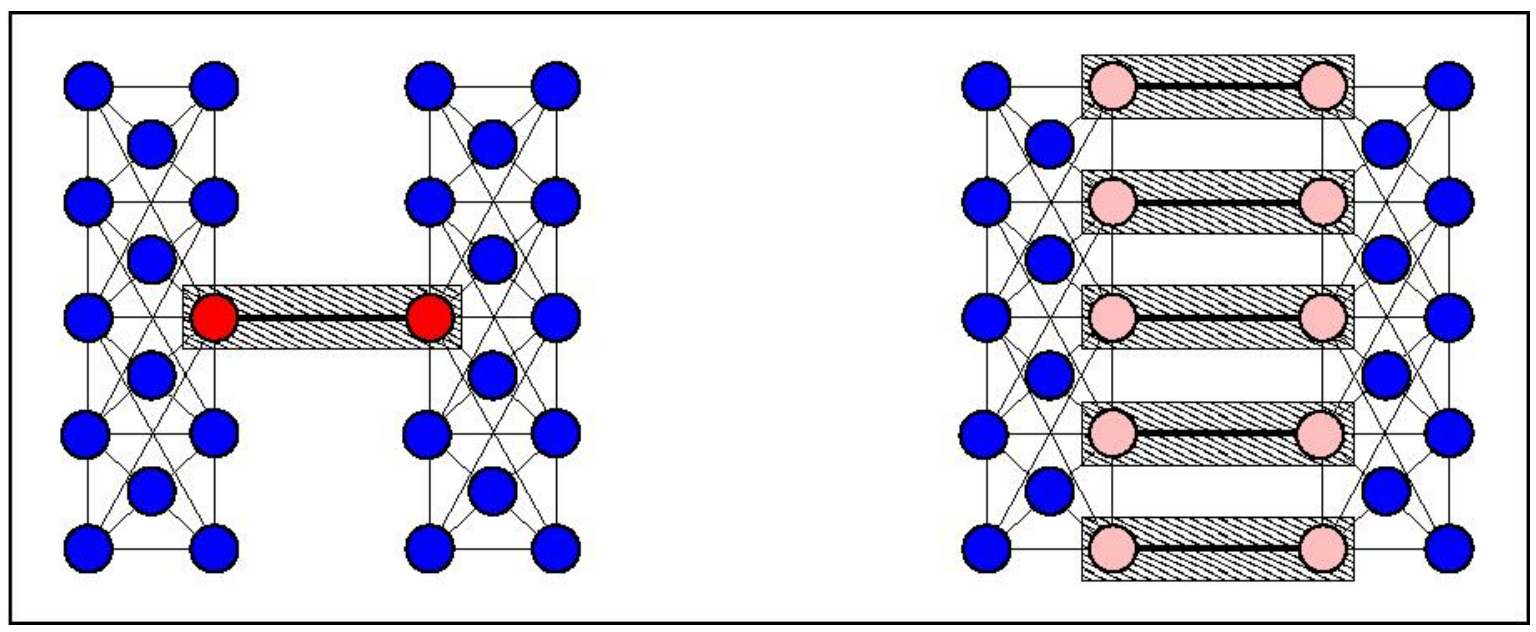

Figure 4. A semantic network where edges bridge knowledge nodes. Blue nodes represent knowledge owned by single agents. Red and pink nodes represent knowledge owned by firms, represented by shaded areas. Left, monopolistic profits accrue to the one firm that owns the only knowledge (red nodes) that bridges between otherwise separate knowledge (blue nodes). Right, smaller profits accrue to many firms that own bridging nodes (pink nodes).

Note that, strictly speaking, knowledge ownership does not pertain to knowledge networks. Indeed, in order to represent both cognitive distance and knowledge ownership we implicitly combined knowledge networks and social networks. In Figure 4, the knowledge network appears with its links and nodes, whereas a social network provided information to draw the shaded areas representing knowledge ownership.

Knowledge ownership does matter for the viability of business models. For instance, the right side of Figure (4) depicts a situation where a number of firms are able to bridge between otherwise disconnected knowledge, drawing profits towards zero. This is in sharp contrast to the monopolistic situation illustrated on the left side. Unfortunately, this aspect is missing from Thompson's definition of mediating technologies.

Thompson (1967) defined mediating technologies with respect to populations of actors. Disregarding any idiosyncratic knowledge, Thompson defined mediating technologies as operating "(...) in standardized ways, and extensively; e.g., with multiple clients or customers distributed in time and space." (Thompson, 1967: 16). Thompson's examples for organizations based on mediating technologies were commercial banks, insurance companies, telephone utilities and postal services. In the 1960s, in many countries around the world utilities used to be State monopolies, whereas banking and insurance were strongly regulated sectors. In that setting it made sense for Thompson to stress standardization: "The commercial bank must find and aggregate deposits from diverse depositors; but however diverse the depositors, the transaction must conform to standard terms $(\ldots)$. It must also find borrowers; but no matter how varied their needs or desires, loans 
must be made according to standardized criteria and on terms uniformly applied to the category to the particular borrower." (Thompson, 1967: 16-17). Today, standardization of procedures is still important, but differentiating from competitors is at least just as important.

Nowadays, organizations that operate on mediating technologies differentiate from one another by certain relevant pieces of knowledge. For instance, banks still standardize their procedures for receiving deposits and granting loans, but differentiate from competitors in details that aim at capturing and fidelizing specific customers. This is particularly evident for merchant banks and even more so for venture capitalists who specialize in specific technologies, but even in Thompson's times small banks operating in local areas adapted their decisions to informal knowledge of investment prospects (Ergungor, 2010).

In any case, this pattern appears to be really prominent today. Bridging is no longer enough, differentiating from competitors by means of difficult-to-imitate knowledge pillars is key. It is the case of Echelon exploiting its expertise with PLC, but generally speaking this reasoning applies equally well to search engines employing unique algorithms, banks developing unique expertise to classify prospective borrowers, insurance companies competing on difficult-to-assess environmental risks, and so on. All too often, bridging alone does not suffice to ensure a competitive advantage. Building up a pillar of unique knowledge is often the feature that makes a particular bridge preferable to those offered by competitors.

Figure 5 illustrates this state of affairs by means of a knowledge network enriched by the indication of which knowledge one particular firm owns. On the left, only one firm owns a pillar made of several red nodes that provides unique knowledge to a bridge that communicates with a set of blue nodes. On the right, one firm owns valuable pillars at both ends of the bridge. The idea is that these firms have a competitive advantage over those that must extract value from a bridge that does not insist on solid pillars.

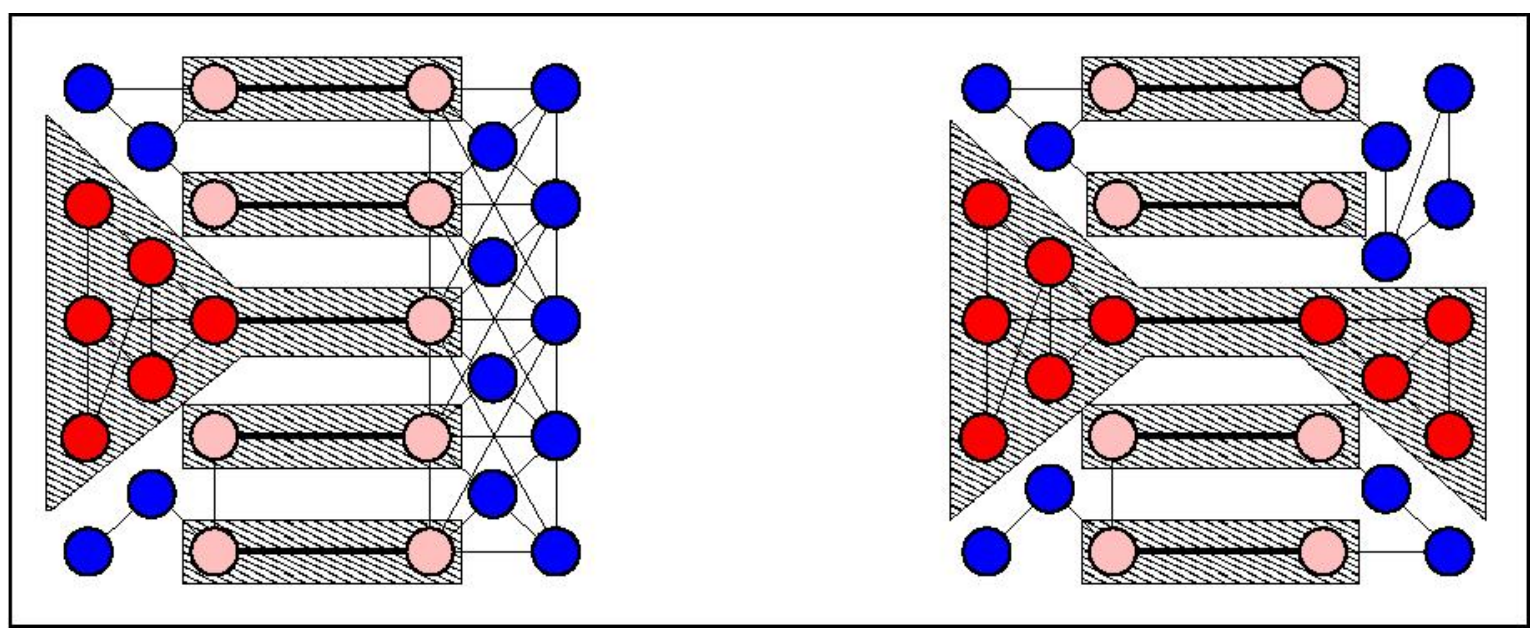

Figure 5. Complex pillars supporting a bridge. Left, a pillar of red nodes that are linked to one another because they interpret knowledge in non-trivial, difficult-to-imitate ways. Right, two such pillars support both edges of a bridge. Either case, the one firm owning at least one such pillars is likely to have a competitive advantage over those that do not.

\section{Discussion}

Thompson wrote his definitions in the 1960s, at a time when markets were booming with standardized goods and profits accrued to those who were quick to exploit increasing returns 
to scale. In that context, niche markets used to be irrelevant and idiosyncratic knowledge was eventually disregarded and downplayed.

By contrast, today we speak of a "knowledge-based economy" precisely because profits typically accrue from rare knowledge rather than large investments in physical assets. Correspondingly, adapting the concept of mediating technologies requires specifying what knowledge firms own, and how their knowledge impacts on their relations with other actors.

This fundamental transformation calls for complex theories of social and cognitive interactions for the generation and valuation of innovations, such as Actor Network Theory (Latour, 1988) or Scaffolding Structures for generative relationships (Lane and Maxfield, 2005). However different from one another, these theories lay stress on relationships and power games between social actors who are described with all of their cognitive prejudices, schemes and limitations. According to both theories, the structure of social networks is paramount for the generation and success of innovations.

Our bridge-pillars interpretive framework may be understood as a simplified, impoverished version of these theories where only bridge-pillars social structures have been considered, which have been further assumed to capture all possible power games. Furthermore, all cognitive processes of generation and alignment of meaning have been assumed to occur in the pillars only.

Simplifications of such a magnitude make sense insofar they allow to capture certain widespread social phenomena in a crude but still effective way. In particular, out of our scheme we propose two propositions to qualify Thompson's concept of mediating technologies. The first one derives straightforwardly from our analysis of the Echelon case-study:

\section{P1: When a mediating technology that bridges between otherwise disconnected popu- lations is easy to imitate, firms should strengthen the pillars of knowledge on which it rests.}

Since at least the "New Economy" bubble, the business press is very much concerned with the extreme likelihood with which e-businesses fail (Rangan and Adner, 2001; Rovenport, 2003). More recently, business analysts have warned that the Internet, by connecting everyone to anyone else, may increase competition to the point of squeezing the profits of all but one player (Bughin et al., 2018). Others have suggested that digitalization should be tied to the traditional core business in order to be a success (Davenport and Westerman, 2018).

We suggest that our Proposition P1 effectively captures what practitioners are observing in a more general and concise way. According to our interpretation, so many e-businesses fail because the Internet makes it all too easy to bridge otherwise disconnected items. However, by making use of some core knowledge pillar that are difficult to imitate - just like PLC for Echelon - businesses can revert the threat of competitive bridging.

Notably, difficult-to-imitate knowledge pillars may make bridging even easier, but only to those who own those pillars. For instance, Echelon arguably extended the range of IloT applications by making use of PLC. In a similar vein, we may remark that in principle the Internet made it easy for anyone to bridge between businesses and consumers (B2C), but Amazon was able to get a paramount position in $\mathrm{B} 2 \mathrm{C}$ by building strong and unassailable knowledge pillars based on their "one click" software and cloud computing platform (AWS). Interestingly, these technological pillars made B2C-bridging even easier, attracting a number of sellers who boosted their offerings.

In general, the knowledge pillars on which mediating technologies rest are generally not mediating technologies themselves. This was clearly the case of Echelon insofar it reinforced its mediating technology with knowledge about PLC, but it applies equally well to search engines caring about algorithms or venture capitalists who specialize into narrowly defined industries. 
Our second proposition originates from the observation that pillars are made of stable, strong ties whereas bridges are made of occasional weak ties. For instance, in our case-study Echelon installed IloT solutions to many different customers, each time establishing a new dedicated bridge between its expertise with physical devices and its expertise with smart software, whereas its expertise with PLC, WC and the other elements of its knowledge pillars are likely to stay there for a long time.

Weak ties come and go. By contrast, strong ties glue the competences of organizations to one another. In order to stress this difference we dare to advance the following proposition:

P2: Since bridges are ephemeral in comparison to knowledge pillars, destruction of a pillar is likely to bear more substantial disruptions than occasional destruction of a bridge.

We illustrate Proposition P2 with an example from banking, a typical mediating technology according to Thompson (1967) which is undergoing profound changes nowadays. Aside from the relatively marginal change of adding online facilities to physical counters, banking is being heavily impacted by the possibility of assessing credit worthiness by applying machine-learning algorithms to big data. In fields such as consumer credit and mortgages, the so-called Fin Tech companies are providing cheaper and more reliable estimators of default rates than bank officials themselves. Moreover, once the FinTech companies have crowded out the market of the best loans, banks are being left with the riskiest customers (Leishon and Thrift, 1999; Wang, 2018).

Possibly, banks and FinTech companies may co-exist if machine learning algorithms will be used as a first screening device only, eventually followed by man-powered analyses (Flögel and Beckamp, 2020). However, a far more destructive threat is posed by the fact that a few digital retailers such as Amazon or Alibaba have privileged access to big data on consumer choices, which they might use to provide mortgages and consumers loans. Currently, this is impossible because of anti-trust legislation but if this possibility will ever come true, mortgages and consumer credit may become a game for a happy few (Boot et al., 2020).

By contrast, banks are generally expected to survive in a niche provided by merchant banking. In particular, lending to small and medium-sized firms is largely based on "soft" factors that can only be assessed by local bankers who engage in long-term relationships with their clients (Ergungor, 2010; Engelberg, Gao and Parsons, 2012; Bird and Knopf, 2015). Moreover, at least medium-sized firms could resort to specialized services to game the Fin Tech algorithms, a circumstance that is likely to shield merchant banks from competitors based on big data (Rona-Tas and Hiss, 2010).

We submit that Proposition P2 captures and generalizes this pattern. Indeed, P2 suggests to trace a distinction between Internet Banking, which opens up new possibilities for bridging between lenders and borrowers, and FinTech, which jeopardizes the very knowledge upon which banks base their ability to bridge. Internet Banking may contribute to the relative success of specific banks, but it does not destroy the banking industry. By contrast, FinTech attacks the pillar of idiosyncratic knowledge on which efficient lending rests. Drawing a parallel with Echelon's expertise with PLC, banks are likely to survive to the extent that their traditional and unique expertise with relational lending will be indispensable in at least one market niche.

\section{Conclusions}

Qualitative research has the purpose of uncovering constructs that are not self-evident, or that have not been adequately conceptualized by available theories. In our case, close examination of an IloT pioneer led us to an extension of Thompson's concept of mediating technologies in order 
to account for the features of today's knowledge economy.

We observed a pattern that appears in otherwise heterogeneous industries, where the Internet or other ICTs can lower the costs of exercising mediation to a point where generalized competition risks to set in. In spite of this dramatic scenario, it is often possible for firms to exploit some specific, difficult-to-imitate knowledge in order to differentiate their mediation services from one another. On the whole, developing a competitive advantage depends on the ability to build pillars of difficult-to-imitate knowledge rather than bridges to exercise mediation.

Traditionally, mediating technologies used to generate profits by enabling their owners to bridge two otherwise separate populations. Since in many instances ICTs drive such profits down to zero, profits are eventually generated by difficult-to-imitate pillars of knowledge on which bridging activities rest. The degree of the ensuing imperfect competition, oligopoly or even monopoly depends on the relevance and uniqueness of knowledge pillars alone. Bridges that rest on those pillars are valuable, the others are not.

Possibly, our point is even more general. Nowadays, globalization processes are making many businesses feel the pressure of competitors from which they used to be shielded by sheer geographical distance, legal arrangements and transportation costs. Even industries that used to be characterized by the long-chain technologies of managing production and exploiting returns to scale are increasingly concerned with the mediating technologies of arranging supply chains, distribution and marketing. Reaching distant markets has become easier in many respects, triggering a game where everyone can compete, but in the limit only a few oligopolists can survive. In the economy at large just like in the industries that have been most deeply affected by ICTs, greater ease of bridging between producers and consumers is for most businesses a risky game. Knowledge pillars become paramount, and quite often only a few actors own the valuable ones.

However, many novel market niches are emerging from ever-increasing consumer variety and sophistication. Greater diversity of goods and services provides opportunities for SMEs to thrive in small or tiny market niches, worldwide. Pillars of unique, difficult-to-imitate knowledge are key to create and exploit such market niches, exchanging geography-based market fragmentation with a knowledge-based market fragmentation that can still permit the existence of a diverse economy where entrepreneurship is the rule, instead of a homogeneous world economy where any market can be reached, and therefore only a few players can survive.

With all of its generality, our interpretive framework comes with a tool - semantic networks that is meant to ease semi-quantitative analysis. Our hope is that in future research the structure of organizational knowledge will be analyzed with respect to its ability to provide nearly-unique pillars for bridging across concepts and their owners. Such findings may stimulate reflection, and they may be possibly embedded in expert systems. However, we did not come up with a simple recipe. Our bridges-and-pillars framework does not automatically provide solutions.

\section{Acknowledgements}

We wish to thank Christoph Broennimann, Chris Dingley, Robert Dolin, David Lane, Robert Maxfield, Sohrab Modi and Ronald Sege for help, encouragement and advice.

\section{Note}

This paper is an improved version of the working paper: "Fioretti, Guido and Kwan, Stephen K. and Niciforo, Stefania (2018): A Business Model Bridging Knowledge Gaps". Available at: https://mpra.ub.uni-muenchen.de/id/eprint/83936 


\section{References}

Bird, R.C., \& Knopf, J.D. (2015). The Impact of Local Knowledge on Banking. Journal of Financial Services Research, 48(1), 1-20.

Boot, A., Hoffmann, P., Laeven, L., \& Ratnovski, L. (2020). Financial Intermediation and Technology: What's old, what's new? ECB Working Paper No. 2438. Frankfurt, European Central Bank.

Bughin, J., Catlin, T., Hirt, M., \& Wilmott, P. (2018). Why Digital Strategies Fail. McKinsey Quarterly, January.

Burt, R.S. (1992). Structural Holes. Cambridge (MA): Harvard University Press.

Casalini, A., Fioretti, G., \& Pyka, A. (2016). Playfulness, Ideology and the Technology of Foolishness in the Creation of a Novel Market Niche for Distributed Control: The case of iPLON. Journal of Organizational Design, 5(6).

Claro Partners (2014). A Guide to Succeeding in the Internet of Things. Accessed $10^{\text {th }}$ September 2019. www.claropartners.com.

Davenport, T.H., \& Westerman, G. (2018). Why So Many High-Profile Digital Transformations Fail. Harvard Business Review, March $9^{\text {th }}$.

Dhanorkar, S. (2019). Environmental Benefits of Internet-Enabled C2C Closed-Loop Supply Chains: A quasi-experimental study of Craiglist. Management Science, 65(2), 660-680.

Dolin, R. (2015). Interview. In Niciforo S: Business Models and Economic Implications of Distributed Control in the Internet of Things Area. University of Bologna, Scuola di Economia, Management e Statistica, Master Thesis.

Echelon (1990). Dec 5, 1990: The Day That Everything Began to Work Together. Company Video.

Echelon (2014). Echelon Expands Presence in Outdoor Lighting with Acquisition of Established Player, Lumewave. Press Release.

Engelberg, J., Gao, P., \& Parsons, C.A. (2012). Friends with Money. Journal of Financial Economics, 103(1), 169-188.

Ergungor, O.E. (2010). Bank Branch Presence and Access to Credit in Low- to Moderate-Income Neighborhoods. Journal of Money, Credit and Banking, 42(7), 1321-1349.

Evans, G.L. (2013). A Novice Researcher's First Walk Through the Maze of Grounded Theory: Rationalization for Classical Grounded Theory. The Grounded Theory Review, 12(1), 37-55.

Fleisch, E., Weinberger, M., \& Wortmann, F. (2014). Business Models and the Internet of Things. Accessed May 22th 2019. www.iot-lab.ch/?page_id=10543.

Flögel, F., \& Beckamp, M. (2020). Will FinTech Make Regional Banks Superflous for Small Firm Finance? Observations from soft information-based lending in Germany. Economic Notes, 49, e12159.

Kemp, R., Schot, J., \& Hoogsma, R. (1998). Regime Shifts to Sustainability Through Processes of Niche Formation: The approach of strategic niche management. Technology Analysis \& Strategic Management, 10(2), 175-195.

Glaser, B.G. (1992). Basics of Grounded Theory Analysis. Mill Valley: Sociology Press. 
Glaser, B.G. (2002). Conceptualization: On theory and theorizing using grounded theory. International Journal of Qualitative Methods, 1(2), 23-38.

Glaser, B.G., \& Holton, J. (2004). Remodeling Grounded Theory. The Grounded Theory Review, 4(1), 1-24.

Glaser, B.G., \& Strauss, A.L. (1967). The Discovery of Grounded Theory. New Brunswick: Aldine Transaction.

Hannan, M.T., \& Freeman, J. (1984). Structural Inertia and Organizational Change. American Sociological Review, 49(2), 303-313.

Lane, D.A., \& Maxfield, R.R. (2005). Ontological Uncertainty and Innovation. Journal of Evolutionary Economics, 15(1), 3-50.

Lane, D.A., \& Maxfield, R.R. (2009). Building a New Market System: Effective action, redirection and generative relationships. In D. Lane, S. van der Leeuw, D. Pumain, \& G. West (Eds.), Complexity Perspectives in Innovation and Social Change, (pp. 263-288). Berlin-Heidelberg: Springer Verlag.

Latour, B. (1988). The Pasteurization of France. Cambridge (MA): Harvard University Press.

Leishon, A., \& Thrift, N. (1999). Lists Come Alive: Electronic systems of knowledge and the rise of credit-scoring in retail banking. Economy and Society, 28(3), 434-466.

Lumpkin, G.T., \& Dess, G.G. (2004). E-Business Strategies and Internet Business Models: How the Internet Adds Value. Organizational Dynamics, 33(2), 161-173.

Nooteboom, B. (2000). Learning and Innovation in Organizations and Economies. Oxford: Oxford University Press.

Orton, J.D. (1997). From Inductive to Iterative Grounded Theory: Zipping the gap between process theory and process data. Scandinavian Journal of Management, 13(4), 419-438.

Rangan, S., \& Adner, R. (2001). Profits and the Internet: Seven Misconceptions. MIT Sloan Management Review, 42(4), 44-53.

Rona-Tas, A., \& Hiss, S. (2010). The Role of Ratings in the Subprime Mortgage Crisis: The art of corporate and the science of consumer credit rating. In M. Lounsbury, \& P.M. Hirsch (Eds.), Markets on Trial: The Economic Sociology of the U.S. Financial Crisis: Part A (pp. 115-155). Bingley: Emerald Group Publishing Limited.

Rossi, F., Bertossi, P., Gurisatti, P., \& Sovieni, L. (2009). Incorporating a New Technology into Agent-Artifact Space: The case of control automation in Europe. In D.A. Lane, S. van der Leeuw, D. Pumain, G. West (Eds.), Complexity Perspectives in Innovation and Social Change (pp. 289-310). Berlin-Heidelberg: Springer Verlag.

Rovenport, S. (2003). Explaining the E-Commerce Shakeout: Why Did So Many Internet-Based Businesses Fail? E-Service Journal, 3(1), 53-76.

Sowa, J.F. (1992). Semantic Neworks. In S.C. Shapiro (Ed.), Encyclopedia of Artificial Intelligence. New York: Wiley.

Suddaby, R. (2006). From the Editors: What Grounded Theory is not. Academy of Management Journal, 49(4), 633-642. 
Tatò, F. (2016). Interview. In R. Cartolano: The Evolution of Organizations Through Change and Continuity: The EcheLON Case. University of Bologna, Scuola di Economia, Management e Statistica, Master Thesis.

Teece, D.J. (2010). Business Models, Business Strategy and Innovation. Long Range Planning, 43(2-3), 172-194.

Thoma, G. (2017). Commercialization Strategy of a General Purpose Technology. In G. Thoma (Ed.), Patent Management and Valuation (pp. 151-178). New York: Routledge.

Thompson, J.D. (1976). Organizations in Action. New York: McGraw-Hill.

Walker, D., \& Myrick, F. (2006). Grounded Theory: An exploration of process and procedure. Qualitative Health Research, 16(4), 547-559.

Wang, J.C. (2018). Technology, the Nature of Information, and Fin Tech Marketplace Lending. Federal Reserve Bank of Boston, Current Policy Perspectives No. 18-3.

Wired (1993). If Your Toaster Had a Brain. Wired, 01-01-1993. Accessed 13 ${ }^{\text {th }}$ May 2018. http://www.wired.com/1993/01/toaster.

Yoo, Y., Henfridsson, O., \& Lyytinen, K. (2010). The New Organizing Logic of Digital Innovation: An agenda for information systems research. Information Systems Research, 21(4), 724-735.

Ziegeldorf, J.H., Garcia Morchon, O., \& Wehrle, K. (2013). Privacy in the Internet of Things: Threats and challenges. Security and Communication Networks, 7(12), 2728-2742. 


\section{Biographies}

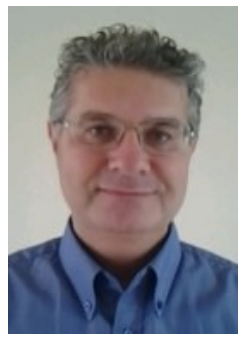

Guido Fioretti. Guido Fioretti, born 1964, graduated in Electronic Engineering and received a $\mathrm{PhD}$ in Economics from La Sapienza University in Rome. He is currently an associate professor of Organization Science at the University of Bologna, Italy. His research interests combine social and cognitive sciences in order to model decision-making facing uncertainty. In particular, he is interested in distributed models of organizational decision-making.

CRediT Statement: Conceptualization, Formal Analysis, Writing - Original Draft, Writing Review \& Editing, Visualization, Project Administration.

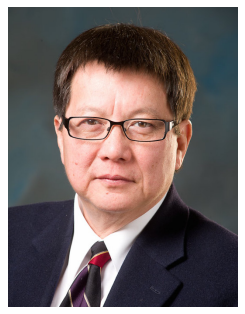

Stephen K. Kwan. Dr. Stephen K. Kwan is Professor Emeritus in the Lucas College and Graduate School of Business at San José State University, and a fellow of the International Society of Service Innovation Professionals (ISSIP). He retired from being the Associate Dean of Graduate Business Programs and Lucas Professor of Service Science. He is still actively involved in research in Service Science, Management, Engineering, and Design (SSMED), global E-Commerce, service standards and standardization. He currently serves on the ISO/IEC JTC1 Sub-Committee 42 Artificial Intelligence, ISO Committee on Consumer Policy, and American National Standards Institute (ANSI)'s Committee on Education, ISO Council, International Policy Advisory Group, and Consumer Interest Forum.

CRediT Statement: Methodology, Resources, Writing - Review \& Editing, Supervision.

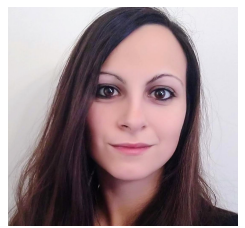

Stefania Niciforo. Stefania Niciforo, born 1988, graduated in Business Administration. She received a fellowship in order to write her MSc thesis in Silicon Valley where she interviewed prominent managers, collected information on strategies related to big data and loT, and attended conferences such as IEEE SIIT 2015, IBM Watson, Samsung Sami and Google loT Robotic. She is currently working as Head of Account Management at Instal, an IT company focusing on high-innovation projects.

CRediT Statement: Conceptualization, Investigation, Data Curation, Funding Acquisition. 